\title{
Erratum to: Clinical characteristics and outcomes of spontaneous bacterial peritonitis caused by Enterobacter species versus Escherichia coli: a matched case-control study
}

Seongman Bae', Taeeun Kim¹, Min-Chul Kim¹, Yong Pil Chong', Sung-Han Kim¹, Heungsup Sung ${ }^{3}$, Young-Suk Lim², Sang-Oh Lee', Mi-Na Kim³, Yang Soo Kim', Jun Hee Woo ${ }^{1}$ and Sang-Ho Choi ${ }^{\text {** }}$

\section{Erratum description}

n.b. The errors and associated corrections described in this document concerning the original manuscript were accountable to the production department handling this manuscript, and thus are no fault of the authors of this paper.

In the original publication of this article [1], the tables were inadvertently omitted. These can be found below:

\footnotetext{
Author details

${ }^{1}$ Department of Infectious Diseases, Asan Medical Center, University of Ulsan College of Medicine, Seoul, Republic of Korea. ${ }^{2}$ Department of Gastroenterology, Asan Medical Center, University of Ulsan College of Medicine, Seoul, Republic of Korea. ${ }^{3}$ Department of Laboratory Medicine, Asan Medical Center, University of Ulsan College of Medicine, Seoul, Republic of Korea.
}

Received: 9 June 2016 Accepted: 13 September 2016 Published online: 28 September 2016

\section{Reference}

1. Bae S, et al. Clinical characteristics and outcomes of spontaneous bacterial peritonitis caused by Enterobacter species versus Escherichia coli: a matched case-control study. BMC Infectious Diseases. 2016;16:252. doi:10.1186/s12879-016-1595-y.

\footnotetext{
* Correspondence: sangho@amc.seoul.kr

'Department of Infectious Diseases, Asan Medical Center, University of Ulsan College of Medicine, Seoul, Republic of Korea

Full list of author information is available at the end of the article
} 
Table 1 Demographic and baseline clinical characteristics of patients with spontaneous bacterial peritonitis

\begin{tabular}{|c|c|c|c|}
\hline Variable & $\begin{array}{l}\text { Enterobacter SBP } \\
(n=32)\end{array}$ & $\begin{array}{l}\text { E. coli SBP } \\
(n=128)\end{array}$ & $P$ \\
\hline Male sex & $26(81.3)$ & $104(81.3)$ & \\
\hline Age, years (mean $\pm S D$ ) & $55 \pm 10.9$ & $55 \pm 11.7$ & \\
\hline SBP subtype & & & 0.03 \\
\hline Definite SBPa & $26(81.2)$ & $78(60.9)$ & \\
\hline Probable SBP & $6(18.8)$ & $50(39.1)$ & \\
\hline Place of acquisition & & & $<0.001$ \\
\hline Community & $12(37.5)$ & $98(76.6)$ & \\
\hline Hospital & $20(62.5)$ & $30(23.4)$ & \\
\hline $\begin{array}{l}\text { Concomitant hepatocellular } \\
\text { carcinoma }\end{array}$ & $21(65.6)$ & $48(37.5)$ & 0.004 \\
\hline Causes of liver cirrhosis & & & 0.79 \\
\hline Hepatitis B virus & $21(65.6)$ & $88(68.8)$ & \\
\hline Hepatitis C virus & $2(6.2)$ & $13(10.2)$ & \\
\hline Alcoholism & $6(18.8)$ & $19(14.8)$ & \\
\hline Others & $3(9.4)$ & $8(6.2)$ & \\
\hline Child-Pugh class & & & 0.006 \\
\hline A & $1(3.1)$ & $0(0)$ & \\
\hline B & $10(31.3)$ & $17(13.3)$ & \\
\hline C & $21(65.6)$ & $111(86.7)$ & \\
\hline MELD score, median (IQR) & $19(15-24)$ & $23(18-29)$ & 0.03 \\
\hline \multicolumn{4}{|l|}{ Underlying diseases } \\
\hline Diabetes mellitus & $9(28.1)$ & $25(19.5)$ & 0.29 \\
\hline Alcoholism & $6(18.8)$ & $23(18.0)$ & 0.92 \\
\hline $\begin{array}{l}\text { Solid cancer (other than } \\
\text { hepatoma) }\end{array}$ & $3(9.4)$ & $4(3.1)$ & 0.14 \\
\hline Chronic kidney disease & $1(3.1)$ & $2(1.6)$ & 0.49 \\
\hline Solid organ transplantation & $1(3.1)$ & $0(0)$ & 0.20 \\
\hline \multicolumn{4}{|l|}{ Comorbid conditions } \\
\hline $\begin{array}{l}\text { Transarterial } \\
\text { chemoembolization } \\
\text { (<30 days) }\end{array}$ & $4(12.5)$ & $4(3.1)$ & 0.052 \\
\hline $\begin{array}{l}\text { Endoscopic intervention } \\
\text { ( } \leq 30 \text { days) }\end{array}$ & $8(25.0)$ & $7(5.5)$ & 0.001 \\
\hline Varix control & 5 & 6 & \\
\hline ERCP & 3 & 1 & \\
\hline $\begin{array}{l}\text { Systemic anticancer } \\
\text { chemotherapy ( } \leq 30 \text { days) }\end{array}$ & $3(9.4)$ & $0(0)$ & 0.007 \\
\hline $\begin{array}{l}\text { Prior hospitalization } \\
\text { ( } \leq 90 \text { days) }\end{array}$ & $26(81.2)$ & 74 (57.8) & 0.01 \\
\hline $\begin{array}{l}\text { Prior antimicrobial therapy } \\
\text { (<30 days) }\end{array}$ & $19(59.4)$ & $36(28.1)$ & 0.001 \\
\hline
\end{tabular}

Values are $\mathrm{n}(\%)$ unless otherwise indicated. MELD model for end-stage liver disease, $I Q R$ interquartile range

$E R C P$ endoscopic retrograde cholangiopancreatography

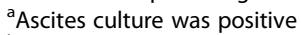

${ }^{\mathrm{b}}$ Ascites culture was negative, while blood culture was positive without any

other primary focus
Table 2 Clinical manifestations and laboratory findings of patients with spontaneous bacterial peritonitis by study group

\begin{tabular}{llll}
\hline Variable & $\begin{array}{l}\text { Enterobacter } \\
\text { SBP } \\
(n=32)\end{array}$ & $\begin{array}{l}\text { E. coli SBP } \\
(n=128)\end{array}$ & $P$ \\
\hline $\begin{array}{llll}\text { Initial clinical manifestation, } \mathrm{n}(\%) \\
\text { Abdominal pain }\end{array}$ & $23(71.9)$ & $90(70.3)$ & 0.86 \\
Fever & $24(75.0)$ & $87(68.0)$ & 0.44 \\
Hepatic encephalopathy & $6(18.8)$ & $34(26.6)$ & 0.36 \\
Septic shock & $5(15.6)$ & $31(24.2)$ & 0.30
\end{tabular}

Upper gastrointestinal bleeding, $9(28.1) \quad 12(9.4) \quad 0.005$
$\mathrm{n}(\%)$

$\begin{array}{llll}\text { Variceal bleeding } & 7 & 11 & \\ \text { Ulcer bleeding } & 1 & 1 & \\ \text { Duodenal invasion of cancer } & 1 & 0 & \\ \text { Concomitant bacteremia, n (\%) } & 11(34.4) & 87(68.0) & 0.001\end{array}$

Laboratory finding, median (IQR)

\begin{tabular}{|c|c|c|c|}
\hline Serum WBC, cells/ML & $\begin{array}{l}8,050 \\
(5,125-13,200)\end{array}$ & $\begin{array}{l}6,450 \\
(4,400-9,675)\end{array}$ & 0.12 \\
\hline Platelets, $\times 10 / \mathrm{mL}$ & $61(44-104)$ & $62(41-85)$ & 0.15 \\
\hline C-reactive protein, $\mathrm{mg} / \mathrm{dL}$ & $6.41(2.1-7.9)$ & $3.06(1.1-6.2)$ & 0.06 \\
\hline Serum creatinine, mg/dL & $1.2(0.9-1.5)$ & $1.2(0.9-1.7)$ & 0.80 \\
\hline Serum bilirubin, mg/dL & $3.8(2.6-12.2)$ & $5.3(3.5-10.3)$ & 0.79 \\
\hline Ascites WBC, cells/uL & $\begin{array}{l}6,160 \\
(1,945-11,795)\end{array}$ & $\begin{array}{l}5,360 \\
(2,690-11,960)\end{array}$ & 0.45 \\
\hline Ascites neutrophils, cells/ $\mu \mathrm{L}$ & $\begin{array}{l}5,058 \\
(1,483-10,899)\end{array}$ & $\begin{array}{l}4,275 \\
(2,092-10,771)\end{array}$ & 0.46 \\
\hline scites protein, mg/dL & $1.5(1.0-2.5)$ & $1.0(0.8-1.4)$ & .003 \\
\hline
\end{tabular}

WBC white blood cells, IQR interquartile range 
Table 3 Antimicrobial susceptibility of isolates of Enterobacter species and Escherichia coli

\begin{tabular}{llll}
\hline Antimicrobial $^{a}$ & $\begin{array}{l}\text { Enterobacter SBP } \\
(n=31)\end{array}$ & $\begin{array}{l}\text { E. coli SBP } \\
(n=125)\end{array}$ & $P$ \\
\hline Cefotaxime & $23 / 31(74.2)$ & $106 / 125(84.8)$ & 0.16 \\
Ceftazidime & $22 / 31(71.0)$ & $106 / 125(84.8)$ & 0.07 \\
Ceftriaxone & $22 / 31(71.0)$ & $106 / 125(84.8)$ & 0.07 \\
$\begin{array}{l}\text { Third-generation } \\
\text { cephalosporins }^{b}\end{array}$ & $22 / 31(71.0)$ & $106 / 125(84.8)$ & 0.07 \\
Cefepime & $25 / 29(86.2)$ & $103 / 123(83.7)$ & 1.00 \\
Ciprofloxacin & $25 / 31(80.6)$ & $76 / 125(60.8)$ & 0.038 \\
Piperacillin/tazobactam & $23 / 31(74.2)$ & $116 / 125(92.8)$ & 0.003 \\
Imipenem & $31 / 31(100.0)$ & $125 / 125$ & 1.00 \\
& & $(100.0)$ & \\
Trimethoprim-sulfamethoxazole & $27 / 31(87.1)$ & $74 / 125(59.2)$ & 0.003 \\
Amikacin & $30 / 31(96.8)$ & $124 / 125(99.2)$ & 0.36 \\
Gentamicin & $27 / 31(87.1)$ & $84 / 125(67.2)$ & 0.03 \\
Tobramycin & $27 / 31(87.1)$ & $81 / 125(64.8)$ & 0.02 \\
\hline
\end{tabular}

Values are $\mathrm{n}(\%)$ unless otherwise indicated

${ }^{a}$ Not all of the isolates underwent susceptibility testing

${ }^{\mathrm{b}}$ Means cefotaxime or ceftriaxone. The susceptibility to third-generation cephalosporins was defined by the breakpoints of the 2008 Clinical Laboratory Standards Institute guidelines (susceptible, $\leq 8 \mu \mathrm{g} / \mathrm{ml}$; intermediate, $16-32 \mu \mathrm{g} /$ $\mathrm{ml}$; and resistant, $\geq 64 \mu \mathrm{g} / \mathrm{ml}$ [ [18]
Table 4 Treatments and outcomes in patients with spontaneous bacterial peritonitis caused by Enterobacter species and Escherichia coli

\begin{tabular}{|c|c|c|c|}
\hline Variable & $\begin{array}{l}\text { Enterobacter } \\
\text { SBP }(n=32)\end{array}$ & $\begin{array}{l}\text { E. coli SBP } \\
(n=128)\end{array}$ & $P$ \\
\hline \multicolumn{4}{|l|}{ Initial empirical antimicrobial agent } \\
\hline Cefotaxime & $27(84.4)$ & $116(90.6)$ & 0.31 \\
\hline Imipenem or meropenem & $4(12.4)$ & $9(7.0)$ & \\
\hline Cefazolin & $1(3.1)$ & $0(0)$ & \\
\hline Ceftazidime & $0(0)$ & $1(0.8)$ & \\
\hline Cefepime & $0(0)$ & $1(0.8)$ & \\
\hline Levofloxacin & $0(0)$ & $1(0.8)$ & \\
\hline Appropriateness of initial therapy & $27(87.1)$ & $109(87.2)$ & 1.00 \\
\hline $\begin{array}{l}\text { Initial response to empirical } \\
\text { treatment }^{\mathrm{a}}\end{array}$ & 26/32 (81.3) & $95 / 117(81.2)$ & 0.995 \\
\hline $\begin{array}{l}\text { Emergence of resistance during } \\
\text { third-generation cephalosporin } \\
\text { treatment }\end{array}$ & $1 / 23(4.3)$ & 0/98 (0) & 0.19 \\
\hline \multicolumn{4}{|c|}{ Duration of hospitalization, median days (IQR) } \\
\hline Overall & $20(11-31)$ & $16(10-26)$ & 0.28 \\
\hline Survivors & $22(12-31)$ & $16(11-26)$ & 0.41 \\
\hline \multicolumn{4}{|c|}{ Duration of antimicrobial use, median days (IQR) } \\
\hline Overall & $15(11-25)$ & $13(8-16)$ & 0.02 \\
\hline Survivors & $16(13-29)$ & $13(10-16)$ & 0.01 \\
\hline ICU care during admission & $2(6.2)$ & $19(14.8)$ & 0.25 \\
\hline \multicolumn{4}{|l|}{ Mortality } \\
\hline 14-day mortality & $7(21.9)$ & $25(19.5)$ & 0.77 \\
\hline 30-day mortality & $12(37.5)$ & 37 (28.9) & 0.35 \\
\hline In-hospital mortality & $12(37.5)$ & $28(21.9)$ & 0.07 \\
\hline
\end{tabular}

Values are $\mathrm{n}(\%)$ unless otherwise indicated. IQR interquartile range, ICU intensive care unit

${ }^{\mathrm{a}}$ Ascitic neutrophil decrease $>25 \%$ observed $48-72 \mathrm{~h}$ after initiating antimicrobials 